\title{
Unmet Personal Patient Needs in Psoriasis Patients with Low Disease Activity on Adalimumab, Etanercept or Ustekinumab
}

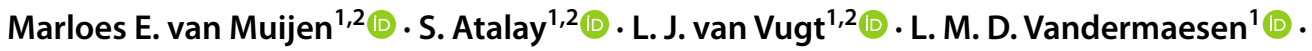 \\ J. M. P. A. van den $\operatorname{Reek}^{1,2}$ (D) E. M. G. J. de Jong ${ }^{1,2,3}$
}

Accepted: 16 January 2021 / Published online: 28 March 2021

(c) The Author(s) 2021

\begin{abstract}
Background Personal treatment goals have been systematically investigated in psoriasis patients with active but not in controlled disease.

Objectives To explore patient needs in psoriasis patients with controlled disease due to biologic therapy with adalimumab, etanercept or ustekinumab.

Methods Treatment needs in patients on adalimumab, etanercept or ustekinumab with a stable low disease activity for $\geq 6$ months and preferably a Psoriasis Area and Severity Index (PASI) $<5$, were explored with the Patient Needs Questionnaire (PNQ). Goal importance was expressed as overall mean importance score, percentage of patients that reported a goal to be quite/very important, and per PNQ subscale. Data were analysed separately for treatment, gender, age group ( $<50$ vs. $\geq 50$ years), biologic naivety and willingness to participate in a pragmatic dose-reduction strategy.

Results Sixty-five patients were included. 'To be free of itching', 'to be healed of all skin defects' and 'to have confidence in the therapy' were rated quite/very important in $78.5 \%$ of the patients, followed by 'to have no fear the disease will progress' $(75.4 \%)$ and 'to get better skin quickly' (75.4\%). Goals related to the subscale 'confidence in healing' were still of high importance in controlled disease. Least importance was attributed towards social goals. For female patients, it was significantly more important than for males to 'feel less depressed' and 'be comfortable showing yourself more in public'. Conclusions Psoriasis patients with controlled disease still report substantial treatment needs, with high importance ascribed to confidence in healing. To apply personalized medicine, treatment needs should be explored on an individual level.
\end{abstract}

\section{Plain Language Summary}

In psoriasis patients, a large reduction in disease severity can lead to a significant improvement in health-related quality of life. In addition to quality-of-life measurements, individual treatment goals can be assessed to evaluate patients' preferences regarding their psoriasis treatment. As opposed to patients with more severe psoriasis, unmet treatment needs in psoriasis patients with stable, low disease activity have barely been reported. In this study, the personal treatment aims of patients with controlled disease due to treatment with adalimumab, etanercept or ustekinumab were explored using the Patient Needs Questionnaire. Sixty-five patients with sustained low disease activity for $\geq 6$ months were included. We found that despite low disease activity, these patients still have substantial patient needs. Patients attributed the highest importance to goals on confidence in healing, in contrast to social goals, which were valued of least importance. For female patients, it was significantly more important to 'feel less depressed' and 'be comfortable showing yourself more in public' compared to male patients. Previous treatment with biologic therapy was not associated with an altered attitude towards specific treatment goals.

\section{Digital Features To view digital features for this article go to https://doi.org/10.6084/m9.figshare.13571201.}

Marloes E. van Muijen

Marloes.vanMuijen@radboudumc.nl

1 Department of Dermatology, Radboud University Medical Centre (Radboudumc), Mailbox 9101, 6500 HB Nijmegen, The Netherlands
2 Radboud Institute for Health Sciences (RIHS), Radboud University Medical Centre (Radboudumc), Nijmegen, The Netherlands

3 Radboud University, Nijmegen, The Netherlands 
Our population with low disease activity seemed to award a lower level of importance to all treatment goals compared to groups of patients with more severe psoriasis that have been described in literature. Since treatment goals differ per patient, individual treatment could be optimized by actively inquiring about the patient's personal treatment goals. Clinicians should be aware that even in patients with controlled disease, substantial personal treatment needs remain.

\section{Key Points}

Despite low disease activity, psoriasis patients with controlled disease due to biologic treatment still have substantial treatment goals.

In this cohort of psoriasis patients with low disease activity, goals related to 'confidence in healing' were considered most important. Social goals were of least importance.

\section{Introduction}

Psoriasis is an immune-mediated chronic inflammatory skin disease that has a significant impact on the patient's quality of life (QoL) [1]. For both dermatologists and patients, reducing the severity of the disease is the main goal of psoriasis treatment [2]. Over the past few years, various targeted biological therapies have become available, enabling a Psoriasis Area of Severity Index (PASI) reduction of $>75 \%$, or even complete remission [3, 4].

It has been reported that a large reduction in the PASI score as a result of biologic therapy can lead to a significant improvement in QoL [5]. However, in a study on 1-year drug survival of adalimumab, etanercept and ustekinumab, we found that $21 \%$ of the patients still reported a Dermatology Life Quality Index (DLQI) > 5 (a moderate or even larger effect on the QoL) after 1 year of biologic treatment [6]. These results indicate that even when patients are in (partial) remission, treatment goals may still continue to exist in this chronic disease. To focus even more on individual patient needs, in addition to QoL, personal treatment goals can be assessed.

In patients with moderate to severe psoriasis and/or nail psoriasis, a wide range of personal treatment goals have already been explored by using the Patient Needs Questionnaire (PNQ) as part of the Patient Benefit Index (PBI) [7-10]. Patient needs in psoriasis patients with sustained controlled disease activity, on the other hand, have rarely been studied. It is important to get insight into the individual treatment goals of this population in order to optimize treatment, improve treatment adherence and facilitate personalized medicine. In the current era, with an expanding arsenal of biologic therapies with the potential to achieve a significant reduction of disease severity, this will become increasingly relevant.

In this study, by means of the PNQ, we investigated the personal treatment aims of psoriasis patients with controlled disease who were using adalimumab, etanercept or ustekinumab in order to get insight into the unmet treatment needs in this population. To date, this has been an under-reported area in psoriasis research.

\section{Materials and Methods}

\subsection{Data Collection and Extraction}

From 22 February 2018, over a time-frame of 1 year, all adult psoriasis patients who were treated with biologics in the Department of Dermatology, Radboud University Medical Centre (Radboudumc), Nijmegen, the Netherlands were screened for a pragmatic dose-reduction strategy in patients with low, stable disease activity on the authorized standard dose of the biologics adalimumab, etanercept or ustekinumab, and invited to participate if eligible. Stable, low disease activity (controlled disease) was defined as low disease activity for at least 6 months according to the treating physician, and preferably a PASI score $\leq 5$ at inclusion. Oral consent was obtained prior to participation. At the start of the dose reduction strategy (baseline), the PNQ was conducted. In addition, PASI scores were determined, and Dermatology Life Quality Index (DLQI) questionnaires were completed by the patients to measure the effect of psoriasis on the health-related quality of life. Patient characteristics were extracted from the prospective BioCAPTURE registry [11, 12], and included gender, age, age at psoriasis onset, body mass index (BMI), co-medication, presence of psoriatic arthritis, previous PASI scores and history of use of a biologic drug. Additionally, the duration of stable disease was calculated as the time the patient had a PASI $\leq 5$ in regular clinic visits prior to the baseline visit. Patients on adalimumab, etanercept or ustekinumab who were eligible for, but refrained from, inclusion in the dose-reduction strategy could volunteer to fill out the PNQ. Since this dosereduction strategy was implemented as part of daily practice, data on patients who denied dose reduction were collected 
anonymously, to ensure these patients that refraining from dose reduction would not influence their psoriasis treatment.

\subsection{Patient Needs Questionnaire}

Treatment needs were explored at baseline using the PNQ [10]. The PNQ validated for psoriasis consists of 25 therapy goals, to which the patients allocate a score ranging from 0 (not important at all) to 4 (very important). As an alternative, 'does not apply to me' is an option, which was valued as a score of 0 in the analyses [13]. Twenty-three out of 25 therapy goals can be assigned to five distinguishable subscales: reducing psychological impairments, reducing social impairments, reducing impairments due to therapy, reducing physical impairments, and building confidence into therapy. Treatment aims 'to get better skin quickly' and 'to regain control of the disease' are not allocated to any subscale [14].

\subsection{Statistical Analysis}

Patient and treatment baseline characteristics were collected and analysed. Goal importance was calculated both as the mean PNQ score per item and the percentage of patients rating a goal 'quite' or 'very' important (=PNQ score 3 or 4). In case of missing PNQ data, importance scores were calculated over the available data. Patient needs were analysed as per biologic, PNQ subscale, gender, age group $(<50$ years vs. $\geq 50$ years), and biologic treatment history. Additionally, anonymous PNQ data on patients who denied dose reduction were compared to the PNQ data of our study population. Data were analysed using descriptive statistics and standard parameters. In comparing continuous outcomes, independent T-tests were performed for parametric variables and Mann-Whitney $U$ tests were performed for non-parametric variables. The threshold of statistical significance was set at $p<0.05$. All statistical analyses were performed using SPSS (Version 25.0, IBM Corp., Armonk, NY, USA).

\section{Results}

Out of 305 patients on biologic therapy who were screened to participate in the dose-reduction strategy, 101 were eligible according to the inclusion criteria. A total of 204 patients were ineligible for dose reduction, due to, for example, uncontrolled disease, the use of biologic agents other than adalimumab, etanercept and ustekinumab, or only recent introduction of the biologics of interest ( $<6$ months ago). Sixty-eight patients out of 101 started dose-tapering, of whom three patients did not complete the PNQ, leaving 65 patients with a stable, low disease activity who completed the PNQ as the main patient group of focus in this study. Out of all eligible patients, 33 decided not to be included for the dose-reduction project, of whom 28 completed the PNQ anonymously. Baseline characteristics of the dosetapering cohort are presented in Table 1. The median PASI score and DLQI were 1.6 and 0.0, respectively, indicating low disease activity and no effect of psoriasis on quality of life. Except for one patient with an absolute PASI score of 6 , all patients had a PASI score $\leq 5$. At inclusion, PASI75 had been reached by 44 patients $(71 \%)$. The median stable disease duration was 2.64 years. The majority of patients were male (68\%). Most patients used adalimumab (55\%), followed by ustekinumab (26\%) and etanercept (19\%). The median BMI was $24.7 \mathrm{~kg} / \mathrm{m}^{2}$. Fourteen patients (22\%) had concomitant psoriatic arthritis.

\subsection{Overall Goal Importance}

Patient needs that were rated most important were 'to get better skin quickly' (mean PNQ score $3.11,95 \%$ confidence interval (CI) 2.75-3.46), 'to be free of itching' (mean PNQ score 3.09, 95\% CI 2.71-3.47) and 'to be healed of all skin defects' (mean PNQ score 3.09, 95\% CI 2.75-3.44). Of least importance were 'to have fewer out-of-pocket-expenses' (mean PNQ score 1.57, 95\% CI 1.14-2.00), 'to be less of a burden to relatives and friends' (mean PNQ score 1.66, 95\% CI 1.24-2.08) and 'to be able to lead a normal working life' (mean PNQ score 1.75, 95\% CI 1.31-2.20) (Fig. 1). The option 'did/does not apply to me' was most often chosen for 'to be able to lead a normal working life' (36.9\%), 'to be able to sleep better' (33.8\%) and 'to have fewer out-of-pocket treatment costs' (30.8\%). Only seven patients reported 'to have no fear that the disease will progress' or 'to be healed of all skin defects' was not applicable, or not important to them.

When goal importance was expressed as the percentage of patients that rated the goal to be quite/very important (PNQ score 3 or 4), 'to be free of itching', 'to be healed of all skin defects' and 'to have confidence in the therapy' were of high importance in $78.5 \%$ of the patients, followed by 'to have no fear the disease will progress' (75.4\%), 'to get better skin quickly' (75.4\%) and 'to regain control of the disease' (72.3\%) (Fig. 2).

The ranking of PNQ items slightly varied when they were ranked by mean importance score rather than the percent of patients that scored a 3 or a 4 for each item. However, the variation in relative rank order did not exceed three places, taking similar ranks as a consequence of identical mean importance scores or the percentage scored as quite/very important into account (Figs. 1 and 2).

Of the five PNQ subscales, most importance was assigned to 'building confidence into therapy' (mean PNQ subscale score $2.85 \pm 1.26$ ). 'Reducing social impairments' was of least importance (mean PNQ subscale score $1.97 \pm 1.36$ ) (Fig. 3). 
Table 1 Patient characteristics

\begin{tabular}{|c|c|c|c|}
\hline Characteristics & $N(\%)$ & Median (range) & Mean \pm SD \\
\hline \multicolumn{4}{|l|}{ Patient characteristics } \\
\hline Gender (male) & $44(68 \%)$ & & \\
\hline Age (years) & & $55(22-79)$ & \\
\hline Age at psoriasis onset (years) & & $23(3-59)$ & \\
\hline Psoriatic disease duration (years) & & $26(6-59)$ & \\
\hline Body mass index $\left(\mathrm{kg} / \mathrm{m}^{2}\right)^{\mathrm{a}}$ & & $24.7(17.07-38.24)$ & \\
\hline Psoriatic arthritis & $14(22 \%)$ & & \\
\hline \multicolumn{4}{|l|}{ Disease activity } \\
\hline PASI at start of current biologic ${ }^{b}$ & & $10.2(2.1-27.4)$ & \\
\hline PASI at start dose-reduction strategy ${ }^{\mathrm{c}}$ & & $1.6(0.0-6.0)$ & \\
\hline DLQI at start dose-reduction strategy & & $0.0(0.0-18.0)$ & \\
\hline Mean duration of stable disease (PASI $\leq 5$ ) at baseline (years) & & & $3.20 \pm 2.62$ \\
\hline Median duration of stable disease (PASI $\leq 5$ ) at baseline (years) & & $2.64(0.00-10.38)$ & \\
\hline Number of patients that had reached PASI75 at inclusion ${ }^{\mathrm{d}}$ & $44(71 \%)$ & & \\
\hline Number of patients that had reached PASI90 at inclusion ${ }^{\mathrm{d}}$ & $27(43.5 \%)$ & & \\
\hline \multicolumn{4}{|l|}{ Treatment at baseline } \\
\hline Adalimumab & $36(55 \%)$ & & \\
\hline Etanercept & $12(19 \%)$ & & \\
\hline Ustekinumab & $17(26 \%)$ & & \\
\hline Dosing of $45 \mathrm{mg}$ every 12 weeks & 12 & & \\
\hline Dosing of $90 \mathrm{mg}$ every 12 weeks & 5 & & \\
\hline \multicolumn{4}{|l|}{ Treatment history } \\
\hline On first biologic treatment at baseline & $30(46 \%)$ & & \\
\hline Previous treatment with one alternative biologic & $29(45 \%)$ & & \\
\hline Previous treatment with two alternative biologics & $2(3 \%)$ & & \\
\hline Previous treatment with three alternative biologics & $2(3 \%)$ & & \\
\hline Previous treatment with four alternative biologics & $2(3 \%)$ & & \\
\hline \multicolumn{4}{|l|}{ Co-medication } \\
\hline Methotrexate/Acitretin & $6(9 \%)$ & & \\
\hline
\end{tabular}

DLQI Dermatology Life Quality Index, PASI Psoriasis Area and Severity index

${ }^{\mathrm{a}} 10$ missing values

${ }^{\mathrm{b}}$ Two missing values

${ }^{\mathrm{c}}$ One missing value

${ }^{\mathrm{d}}$ Three missing values

Differences in treatment goals by type of biologic are shown in Figs. 1 and 2 and described in Online Supplementary Material (OSM) 2.

\subsection{Personal Treatment Goals by Gender}

Analysed by gender, there were no statistically significant differences in treatment goals, except for 'to feel less depressed' (mean PNQ score 2.43 vs. $1.57, \mathrm{p}=0.05$ ) and 'to be comfortable showing yourself more in public' (mean PNQ score 3.10 vs. $2.11, p=0.02$ ), which were rated significantly higher in female patients. Overall, female patients attributed higher scores to all treatment goals than men, except for 'to be able to lead a normal sex life', 'be free of pain', 'need less time for daily treatment' and 'be less dependent on doctor and clinic visits' (Fig. 4).

\subsection{Personal Treatment Goals by Age}

The younger patients $(<50$ years old $)$ assigned higher importance scores to 23 out of the 25 PNQ items. The mean importance scores attributed to 'to be able to lead a normal everyday life', 'to be comfortable showing yourself more in public', 'to regain control of the disease', 'to have fewer side effects' and 'to be able to lead a normal working life' proved significantly higher in the younger patient group (Fig. 5). 
Fig. 1 Patient needs in psoriasis patients with controlled disease sorted according to relative importance and subgrouped by biologic treatment $(N=65)$

Fig. 2 Goal importance in psoriasis patients with controlled disease, sorted according to the percentage of patients that reported each individual treatment goal to be 'quite important' or 'very important' $(N=65)$

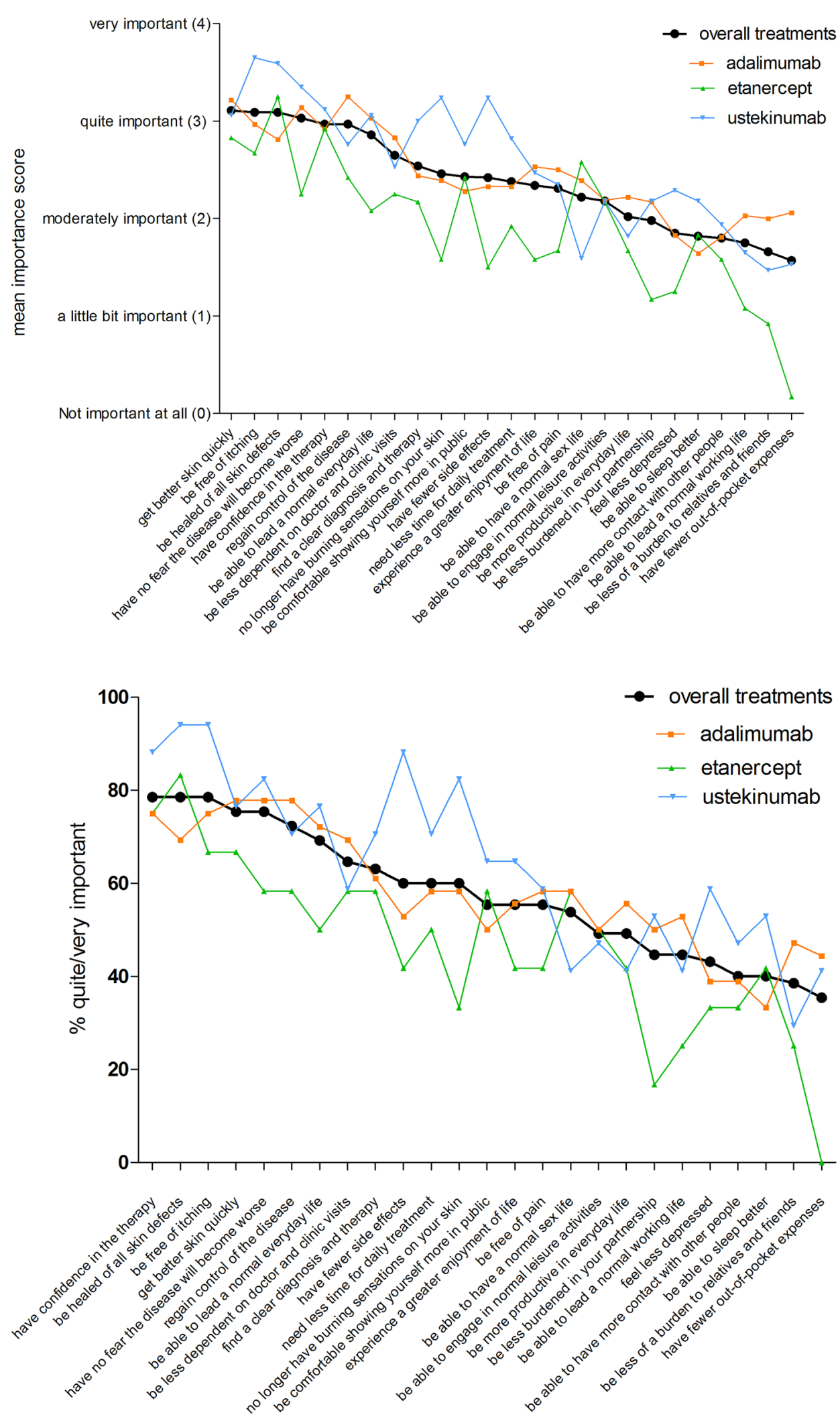




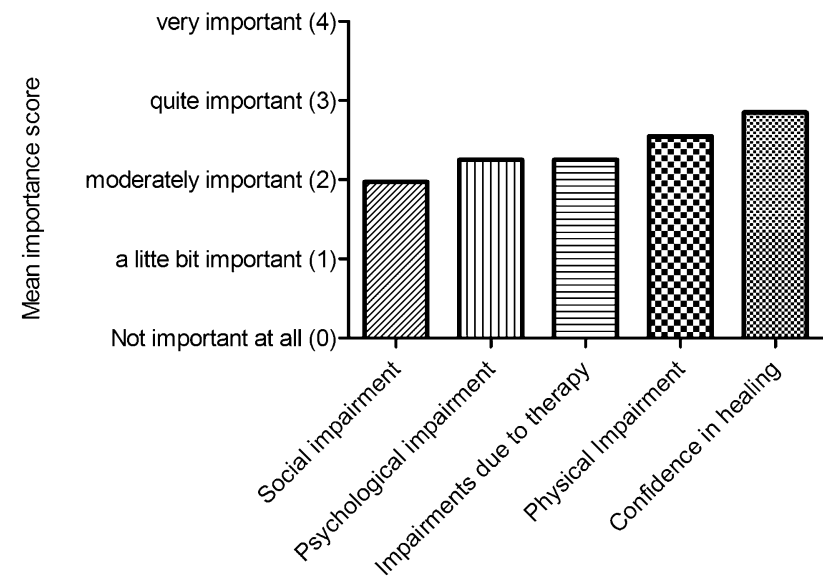

Fig. 3 Mean importance score of treatment goals reported by psoriasis patients with controlled disease $(N=65)$, grouped according to each subscale of the patient needs questionnaire

\subsection{Personal Treatment Goals by Treatment History}

At the start of the dose-reduction strategy, 30 patients were on their first biologic. Of the 35 patients who had previously used biologic therapy, 29 were being treated with their second biologic. Classified for having had biologic treatment in the past, there were no significant differences in treatment needs between patients with a history of biologic use and patients on their first biologic.

\subsection{Personal Treatment Goals by the Willingness to Participate in a Dose-Reduction Strategy}

Twenty-eight patients who did not participate in the dosereduction strategy volunteered to complete the PNQ. Due to incomplete answers, the mean importance score for seven PNQ items was calculated over 27 patients. Since these data had been collected anonymously, baseline characteristics were unavailable. We compared this group to our study population. Analysed for the intent to participate in the dose-reduction strategy, 'to have confidence in therapy' was granted the highest mean importance score (mean PNQ score 3.57, 95\% CI 3.18-3.96) in the group that denied dose reduction, with a statistically significant difference compared to the dose-reduction group (mean 2.97, 95\% CI 2.62-3.32) $(p=0.02)$. All other treatment goals showed no significant differences between both groups.

\section{Discussion}

By means of the PNQ, our study explored personal treatment goals in psoriasis patients with sustained low disease activity for at least 6 months, whilst being treated with etanercept, adalimumab or ustekinumab. We found that despite low disease activity, these patients still have substantial patient needs. Highest importance was attributed to goals on confidence in healing, which was in contrast to social
Fig. 4 Difference in patient needs according to gender in a population with controlled psoriasis $(N=65) \cdot{ }^{*} p \leq 0.05$

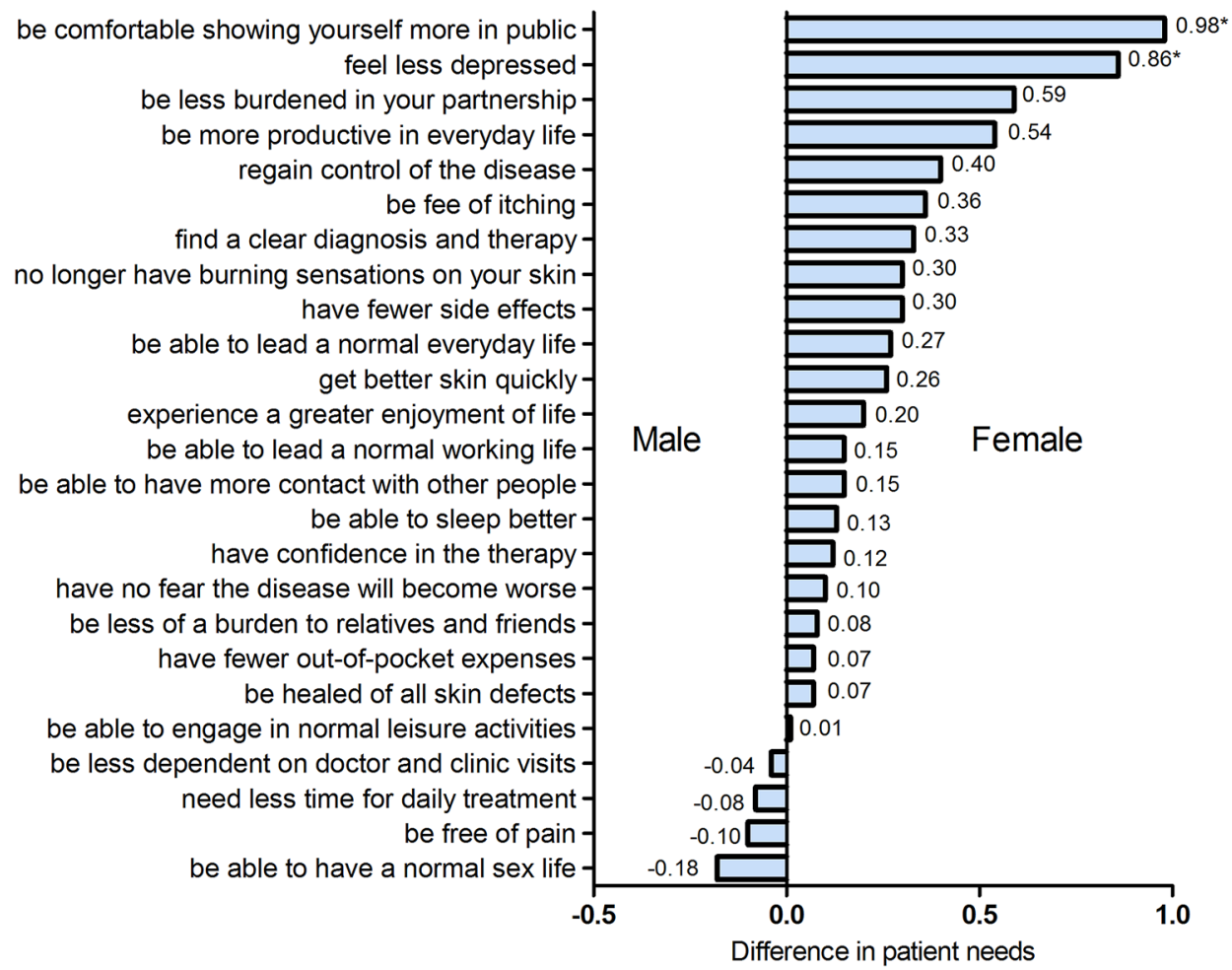


Fig. 5 Difference in patient needs according to age group in a population with controlled psoriasis $(N=65) .{ }^{*} p \leq 0.05$

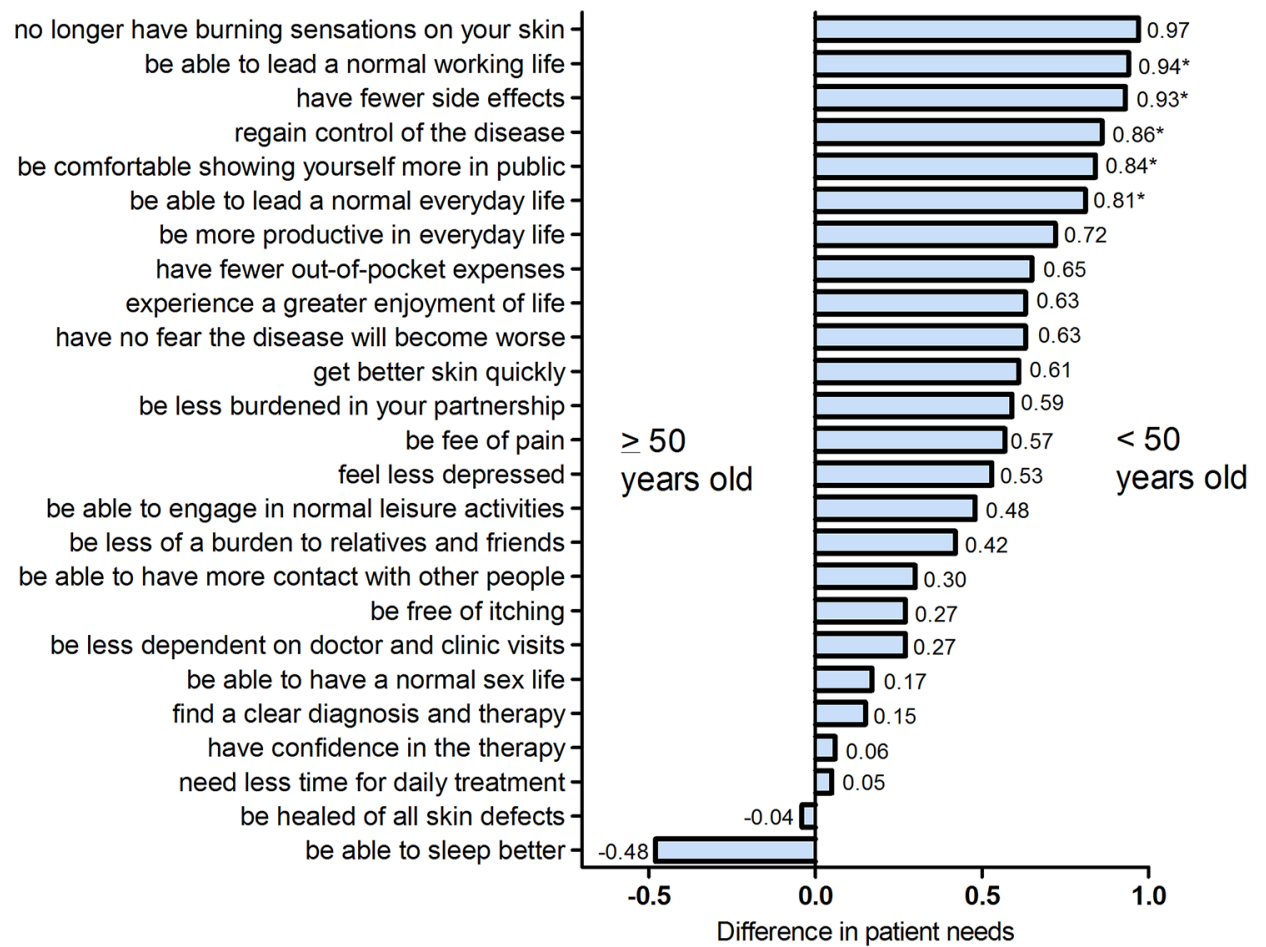

goals, which were valued as being of least importance. For female patients, it was significantly more important to 'feel less depressed' and 'be comfortable showing yourself more in public' compared to male patients. Previous treatment with biologic therapy was not associated with a change in patients' attitude towards specific patient needs. Analysed for the intent to participate in a dose-reduction strategy, 'to have confidence in therapy' was granted the highest mean importance score in the group that denied dose reduction, with a significant difference compared to the dose-reduction group.

Treatment goals 'to have confidence in therapy', 'be healed of all skin defects' and 'be free of itching' were most frequently $(78 \%)$ ranked as 'quite' to 'very' important. As stated, this cohort included patients with low disease activity only. Therefore, high importance scores could imply that certain treatment goals have not yet been reached in this population, or could express the patients' wish that alreadyachieved goals would persist. When compared to a cohort with high disease activity as described by Maul et al. (5343 patients from Germany and Switzerland, mean PASI 14.2 \pm 9.7, DLQI $11.3 \pm 7.2$ ), 'to get better skin quickly' and 'to be healed of all skin defects' were most important to patients, albeit with a higher frequency (94\% ranked it as quite to very important). Of note, the top ten goals with highest importance scores were comparable in both studies. There does not seem to be a large difference between the relative importance of different treatment goals, based on ranking of PNQ items, between patients with stable low disease activity and patients with moderate to severe psoriasis. The widest variation in relative rank order was seen in 'to have fewer out-of-pocket-expenses' (25th in our cohort vs. 16th in Maul et al.), probably due to the differences in healthcare-related costs [15]. To 'be able to have a normal sex life' (16th in our cohort vs. 22nd in Maul et al.) and 'to be able to lead a normal working life' (19th/20th in our cohort vs. 25th in Maul et al.) were the only other items with $>5$ ranks of difference between these two studies. Patients with higher disease activity seem to give higher importance scores to treatment goals compared to patients with low disease activity. The percentages of patients that reported a goal to be quite/very important ranged from $94.0 \%$ for the most important to $49.7 \%$ for the least important item in the study by Maul et al. In our study, however, these percentages were relatively lower, ranging from 78.5 to $35.4 \%$. Nevertheless, even psoriasis patients with relatively low disease activity still have substantial patient needs, which should be considered in daily practice.

Treatment goals 'to feel less depressed' and 'to be more comfortable showing yourself in public' were rated significantly more important by female compared to male patients. This is in line with the findings by Böhm et al., who reported that women perceive a greater negative impact of psoriasis on mental health, self-esteem and maintaining composure in social situations compared to men [16]. Maul et al. and Blome et al. found significant gender differences in patient needs in patients with moderate to severe psoriasis in 20 , respectively 19, out of 25 PNQ items [7, 9]. However, since 
the absolute differences in patient needs scores were relatively small, these findings might be of only minor clinical importance. Nevertheless, it has been reported that female patients are less satisfied with biological treatment for psoriasis [17]. This study suggests that this difference may be independent of the current state of psoriasis, either mild or severe.

Patients under the age of 50 years, compared to those aged $\geq 50$ years, reported higher mean importance scores in 23 out of 25 PNQ items, with significant differences in five items. In both our study and the study on patients with moderate to severe psoriasis by Blome et al. [9], 'to be able to lead a normal working life', 'to be comfortable showing yourself more in public' and 'to have fewer side effects' were rated significantly more important in patients aged $<50$ years. A history of previous treatments with biologic therapy was not associated with an altered attitude towards specific patient needs. However, most patients who had a treatment history with biologics had received only one other type of biologic. It could hypothetically be the case that more extensive treatment histories would lead to differences in PNQ, due to negative experiences in the past.

When comparing our study population (patients motivated to start dose reduction) to the patients who were eligible for but denied dose reduction, we found that 'to have confidence in therapy' was of higher importance in the group that denied dose reduction. This could imply that lack of trust in their current antipsoriatic therapy played a role in their decision not to participate in dose reduction. This finding could be relevant for physicians who want to apply dose-reduction strategies in daily practice.

\subsection{Limitations}

Since this pragmatic dose-reduction strategy was part of daily practice, the preferred cut-off point of a PASI $\leq 5$ for participation was not strictly enforced if patients preferred dose reduction over usual care. We used a PASI $\leq 5$ as part of our definition of low disease activity, based on the prevailing guidelines at the time of inclusion. Currently, treatment goals are shifting towards a PASI score $\leq 3$ [18]. Although an absolute PASI $\leq 3$ was not required in our study, only six patients had a PASI score $>3$ at the start of the dosereduction strategy.

Since patients were asked to complete the PNQ at the start of a dose-tapering project, it is possible that their answers to the PNQ were influenced by any stress or fear of disease progression as a result of the new dosing strategy. However, since all patients volunteered and provided oral consent to start dose tapering, we think this effect was limited.

The PNQ is a subset of the PBI, which was originally designed to evaluate patient-relevant therapeutic benefits longitudinally, by measuring patient needs at baseline (using
PNQ) and the extent to which these needs are achieved after treatment (using the PBQ, Patient Benefit Questionnaire). Although previously applied as such, the PNQ is not formally validated for cross-sectional measurements. Since we did not aim to evaluate treatment benefit, we did not use the PBQ. However, it would have been interesting to assess to what extent the goals in our population had already been reached, or why certain patients had reported a goal to be 'not applicable'. This would facilitate interpretation here. The relatively small sample size and the fact that correction for multiple testing was not performed implies that the results must be interpreted with caution.

In conclusion, psoriasis patients with controlled disease due to biologic treatment still have substantial treatment goals, in an order comparable to patients with more severe psoriasis. They do, however, award their personal treatment goals a lower level of importance compared to patients with more severe disease described in literature. We reported subtle differences in patient needs stratified per gender and age. Clinicians who want to apply dose-reduction strategies should actively inquire about the level of confidence in therapy, as lack of trust in their therapy may exclude eligible patients from dose reduction. Since treatment goals differ per patient, individual treatment could be optimized by actively inquiring about the patient's personal treatment goals. As goal importance is a distinct concept from qualityof-life measurements, addressing both these two items could improve the quality of care [8].

Supplementary Information The online version contains supplementary material available at https://doi.org/10.1007/s40801-021-00227-w.

Acknowledgements We would like to thank all patients who completed the questionnaires, and Marisol Otero, Mascha Eilander, and Lia Schalkwijk for their contribution to the collection and processing of the data.

\section{Declarations}

\section{Funding sources None.}

Conflicts of interest/Competing interest ME van Muijen carries out clinical trials for Abbvie, Celgene, Janssen and Novartis, and has received a speaker's fee from Janssen. All funding is not personal but goes to the independent research fund of the Department of Dermatology of the Radboud University Medical Centre Nijmegen, The Netherlands. S Atalay has received grants from Janssen, Abbvie, Celgene and Novartis and a congress fee from Celgene. All funding is not personal but goes to the independent research fund of the Department of Dermatology of the Radboud University Medical Centre Nijmegen, The Netherlands. LJ van Vugt carried out clinical trials for AbbVie, Celgene, Janssen and Novartis. All funding is not personal but goes to the independent research fund of the Department of Dermatology of the Radboud University Medical Centre Nijmegen, The Netherlands. LMD Vandermaesen has no conflicts to declare. JMPA van den Reek carries out clinical trials for AbbVie, Celgene and Janssen; has received speaker's fees/attended advisory boards for AbbVie, BMS and Jans- 
sen and has received reimbursement for attending a symposium from Celgene and AbbVie. All funding is not personal but goes to the independent research fund of the Department of Dermatology of the Radboud University Medical Centre Nijmegen, The Netherlands. EMGJ de Jong has received research grants for the independent research fund of the Department of Dermatology of the Radboud University Medical Centre Nijmegen, The Netherlands from AbbVie, Pfizer, Novartis, Janssen Pharmaceuticals and Leo Pharma, and has acted as consultant and/or paid speaker for and/or participated in research sponsored by companies that manufacture drugs used for the treatment of psoriasis including AbbVie, Janssen, Novartis, Lily, Celgene, Leo Pharma, UCB and Almirall. All funding is not personal but goes to the independent research fund of the department of Department of Dermatology of the Radboud University Medical Centre Nijmegen, The Netherlands.

Ethical approval Ethics approval was not required as the study was not subject to the Medical Research Involving Human Subjects Act (WMO). All patients who agreed to start dose tapering had been included in the prospective BioCAPTURE registry, and therefore provided written informed consent to use their clinical data for scientific purposes.

Consent to participate All patients provided verbal informed consent.

Consent for publication Not applicable.

Availability of data and material All data generated or analysed during this study are included in this published article and its Supplementary Information files.

Code availability All statistical analyses were performed using SPSS (Version 25.0. Armonk, NY: IBM Corp).

Author's contributions All authors contributed to the study conception and design. Material preparation and data collection was performed by MEM, SA, LJV and LMDV. Analyses were performed by MEM and SA. Supervision was performed by JMPAR and EMGJJ. The first draft of the manuscript was written by MEM and all authors commented on previous versions of the manuscript. All authors read and approved the final manuscript.

Open Access This article is licensed under a Creative Commons Attribution-NonCommercial 4.0 International License, which permits any non-commercial use, sharing, adaptation, distribution and reproduction in any medium or format, as long as you give appropriate credit to the original author(s) and the source, provide a link to the Creative Commons licence, and indicate if changes were made. The images or other third party material in this article are included in the article's Creative Commons licence, unless indicated otherwise in a credit line to the material. If material is not included in the article's Creative Commons licence and your intended use is not permitted by statutory regulation or exceeds the permitted use, you will need to obtain permission directly from the copyright holder. To view a copy of this licence, visit http://creativecommons.org/licenses/by-nc/4.0/.

\section{References}

1. Parisi R, Symmons DP, Griffiths CE, Ashcroft DM. Global epidemiology of psoriasis: a systematic review of incidence and prevalence. J Invest Dermatol. 2013;133(2):377-85. https://doi. org/10.1038/jid.2012.339.

2. Armstrong AW, Siegel MP, Bagel J, Boh EE, Buell M, Cooper KD, et al. From the Medical Board of the National
Psoriasis Foundation: Treatment targets for plaque psoriasis. J Am Acad Dermatol. 2017;76(2):290-8. https://doi.org/10.1016/j. jaad.2016.10.017.

3. Sawyer LM, Malottki K, Sabry-Grant C, Yasmeen N, Wright E, Sohrt A, et al. Assessing the relative efficacy of interleukin-17 and interleukin-23 targeted treatments for moderate-to-severe plaque psoriasis: A systematic review and network meta-analysis of PASI response. PLoS ONE. 2019;14(8):e0220868. https://doi. org/10.1371/journal.pone.0220868.

4. Bai F, Li GG, Liu Q, Niu X, Li R, Ma H. Short-Term Efficacy and Safety of IL-17, IL-12/23, and IL-23 inhibitors brodalumab, secukinumab, ixekizumab, ustekinumab, guselkumab, tildrakizumab, and risankizumab for the treatment of moderate to severe plaque psoriasis: a systematic review and network meta-analysis of randomized controlled trials. J Immunol Res. 2019;2019:2546161. https://doi.org/10.1155/2019/2546161.

5. Mattei PL, Corey KC, Kimball AB. Psoriasis Area Severity Index (PASI) and the Dermatology Life Quality Index (DLQI): the correlation between disease severity and psychological burden in patients treated with biological therapies. J Eur Acad Dermatol Venereol JEADV. 2014;28(3):333-7. https://doi.org/10.1111/ jdv.12106.

6. van den Reek JM, Zweegers J, Kievit W, Otero ME, van Lumig $\mathrm{PP}$, Driessen RJ, et al. "Happy" drug survival of adalimumab, etanercept and ustekinumab in psoriasis in daily practice care: results from the BioCAPTURE network. Br J Dermatol. 2014;171(5):1189-96. https://doi.org/10.1111/bjd.13087.

7. Maul JT, Navarini AA, Sommer R, Anzengruber F, Sorbe C, Mrowietz U, et al. Gender and age significantly determine patient needs and treatment goals in psoriasis - a lesson for practice. J Eur Acad Dermatol Venereol JEADV. 2019;33(4):700-8. https://doi. org/10.1111/jdv.15324.

8. Blome C, Costanzo A, Dauden E, Ferrandiz C, Girolomoni G, Gniadecki R, et al. Patient-relevant needs and treatment goals in nail psoriasis. Qual Life Res. 2016;25(5):1179-88. https://doi. org/10.1007/s11136-015-1136-y.

9. Blome C, Gosau R, Radtke MA, Reich K, Rustenbach SJ, Spehr $\mathrm{C}$, et al. Patient-relevant treatment goals in psoriasis. Arch Dermatol Res. 2016;308(2):69-78. https://doi.org/10.1007/s0040 3-015-1613-8.

10. Augustin M, Radtke MA, Zschocke I, Blome C, Behechtnejad J, Schafer I, et al. The patient benefit index: a novel approach in patient-defined outcomes measurement for skin diseases. Arch Dermatol Res. 2009;301(8):561-71. https://doi.org/10.1007/ s00403-009-0928-8.

11. Zweegers J, van den Reek JM, van de Kerkhof PC, Otero ME, Ossenkoppele PM, Njoo MD, et al. Comparing treatment goals for psoriasis with treatment decisions in daily practice: results from a prospective cohort of patients with psoriasis treated with biologics: BioCAPTURE. Br J Dermatol. 2014;171(5):1091-8. https://doi.org/10.1111/bjd.13137.

12. van den Reek J, Seyger MMB, van Lumig PPM, Driessen RJB, Schalkwijk CJM, Berends MAM, et al. The journey of adult psoriasis patients towards biologics: past and present - Results from the BioCAPTURE registry. J Eur Acad Dermatol Venereol JEADV. 2018;32(4):615-23. https://doi.org/10.1111/jdv.14684.

13. Feuerhahn J, Blome C, Radtke M, Augustin M. Validation of the patient benefit index for the assessment of patient-relevant benefit in the treatment of psoriasis. Arch Dermatol Res. 2012;304(6):433-41. https://doi.org/10.1007/s00403-012-1256-y.

14. Blome C, Augustin M, Behechtnejad J, Rustenbach SJ. Dimensions of patient needs in dermatology: subscales of the patient benefit index. Arch Dermatol Res. 2011;303(1):11-7. https://doi. org/10.1007/s00403-010-1073-0.

15. Pendzialek JB, Simic D, Stock S. Differences in price elasticities of demand for health insurance: a systematic review. Eur J 
Health Econ. 2016;17(1):5-21. https://doi.org/10.1007/s1019 8-014-0650-0.

16. Bohm D, Stock Gissendanner S, Bangemann K, Snitjer I, Werfel T, Weyergraf A, et al. Perceived relationships between severity of psoriasis symptoms, gender, stigmatization and quality of life. J Eur Acad Dermatol Venereol JEADV. 2013;27(2):220-6. https:// doi.org/10.1111/j.1468-3083.2012.04451.x.

17. van der Schoot LS, van den Reek J, Groenewoud JMM, Otero ME, Njoo MD, Ossenkoppele PM, et al. Female patients are less satisfied with biological treatment for psoriasis and experience more side-effects than male patients: results from the prospective BioCAPTURE registry. J Eur Acad Dermatol Venereol JEADV. 2019;33(10):1913-20. https://doi.org/10.1111/jdv.15733.

18. Carretero G, Puig L, Carrascosa JM, Ferrándiz L, Ruiz-Villaverde $\mathrm{R}$, de la Cueva $\mathrm{P}$, et al. Redefining the therapeutic objective in psoriatic patients candidates for biological therapy. J Dermatol Treat. 2018;29(4):334-46. https://doi.org/10.1080/09546 634.2017.1395794. 